\title{
AMERICAN VISCERAL LEISHMANIASIS: A CASE REPORT
}

LANGONI H. (1,2), LUCHEIS S. B. (1), DA SILVA R. C. (1), CASTRO A. P. B. (1), PAES A. C. (1)

(1) Department of Veterinary Hygiene and Public Health, School of Veterinary Medicine and Animal Husbandry (FMVZ), São Paulo State University (UNESP), Botucatu, São Paulo, Brazil; (2) Zoonosis Searches Nucleus (NUPEZO), School of Veterinary Medicine and Animal Husbandry (FMVZ), São Paulo State University (UNESP), Botucatu, São Paulo, Brazil.

ABSTRACT: Visceral leishmaniasis is a zoonotic disease caused by parasites of the Leishmania genus. Dog is the major source of infection to man, especially in urban areas. The authors report a case of visceral leishmaniasis in a pit bull female dog from Bocaina, São Paulo, Brazil. The animal presented clinical signs compatible with leishmaniasis, including skin lesions in the body and partial damage of the external ears. The indirect fluorescent antibody test (IFAT) demonstrated a titer of 1280, and promastigote forms of Leishmania sp were isolated by the culture of bone marrow puncture. Cytological analysis of the lymph node and smear of the bone marrow puncture revealed macrophages containing amastigote forms of Leishmania $\mathrm{sp}$ in their inner region. The test of Polymerase Chain Reaction (PCR) utilized the primers LINR4 and LIN19, which amplify 720 base pairs, specific for Leishmania sp. The authors discuss the importance of techniques for a quick and precise diagnosis to this serious zoonosis with great impact in animal and public health.

KEY WORDS: Leishmania, zoonosis, dog, diagnosis, hemoculture, serology, polymerase chain reaction (PCR).

\section{VIDEOS}

1 - Clinical presentation of the dog. (logo .wmv, logo .swf)

2 - Pre-scapular lymph node puncture. (logo .wmv, logo .swf)

3 - Bone marrow puncture. (logo .wmv, logo .swf)

4 - Promastigote forms isolation in liver infusion tryptose (LIT) medium. (logo .wmv, logo .swf)

CORRESPONDENCE TO: S. B. LUCHEIS, Rodovia Marechal Rondon, Km 279, Caixa Postal 108, 18650-970, São Manuel, São Paulo, Brazil. Email: silucheis@bol.com.br 


\section{INTRODUCTION}

Visceral Leishmaniasis is a zoonotic disease caused by Leishmania chagasi / Leishmania infantum, and the major means of parasite transmission to man and other mammalian hosts is the bite of phlebotomine sandflies of the genera Phlebotomus or Lutzomyia. Lutzomyia longipalpis is the major transmitter of $L$. chagasi in Brazil (10). The disease is endemic in America, as well as in European and Asiatic countries $(13,19)$, and new areas of infection dissemination are being identified $(6,21)$. The fact that dogs are the main urban reservoir of $L$. chagasi makes canine visceral leishmaniasis not only a serious problem in veterinary medicine but also a severe and unsolved problem of public health.

The disease occurrence in a certain area depends fundamentally on the presence of the susceptible vector and the equally susceptible host/reservoir. Originally, American Visceral Leishmaniasis had forest environment as its target, involving mainly wild animals and the Canidae (dogs, wolves, jackals, and foxes). However, changes in the environment, such as climatic alterations, deforestation, human migration from rural to peripheral urban areas, and the bad conditions of dwelling and hygiene, provided too much more cases of the disease.

American Visceral Leishmaniasis has a wide geographic distribution. It is present in 11 countries in South and Central America, including Brazil, where the areas of more prevalence are the Northeast and the Midwest.

The first report on the occurrence of Canine Visceral Leishmaniasis in São Paulo State was in 1998 in Araçatuba (northwest region of the state) and it was diagnosed by the Pathology Service of the Veterinary Hospital, UNESP (14). Since then, the disease is being disseminated along Marechal Rondon Highway, and in Araçatuba region cases of canine transmission are already related in 40 municipalities (8). According to the Epidemiological Vigilance Center, from 1998 to December 2000, 29 human cases of American Visceral Leishmaniasis were notified, with 24 cases in Araçatuba, two in Birigüi, one in Guararapes, and two in Valparaíso; all cities near Araçatuba.

Domestic dogs are important protozoan reservoirs in nature, as well as foxes in the Northeast, Southeast and South of Central Brazil, acting as natural hosts and being responsible for the disease in savage environments. Clinical signs of the disease in dogs may vary from apparently healthy animals, with unapparent infections, to animals presenting different symptoms in several stages of the unapparent disease, 
contributing to its dissemination in case of migration by individuals from endemic to non-endemic areas (3). Generally, symptomatic dogs present fever, anemia, skin lesions, edema, hypergammaglobulinemia, hepatosplenomegaly, and lymphadenopathy, among other signs $(7,18)$.

Direct parasitologic diagnosis of leishmaniasis is the simplest and most utilized too. The essence of this diagnostic test is the observation of amastigote forms of Leishmania $\mathrm{sp}$ in the macrophages cytoplasm or in the intercellular space by histopathological analysis of the bone marrow smear or lymph node aspiration, which is previously fixed in methyl alcohol and then colored by the Giemsa method.

Other broadly utilized parasitological method is the culture of peripheral blood or lymph node and bone marrow puncture material in a specific medium, such as the LIT (Liver Infusion Tryptose) medium, by incubation in a stove at $28-30^{\circ} \mathrm{C}$ with evaluations made in a 1000X fiber optic microscope every 15 days for four months. Among the serological techniques, the indirect fluorescent antibody test (IFAT) is the most utilized. Its sensitivity ranges from $90 \%$ to $100 \%$ and the specificity is about $80 \%$ for serum samples $(12,17)$. It is a sensitive technique, nevertheless, with cross reactions possibility, especially between Chagas' disease and American Cutaneous Leishmaniasis (16). In the case of Visceral Leishmaniasis, high levels of serum antibodies are generally observed, approximately more than 1:80 for serum samples, and titers lower than this would need a new sample for confirmation by serology and other diagnostic methods (9). A suitable obtainment of the material for hemoculture or lymph node and bone marrow puncture to isolate the agent, including total asepsis in the laboratory, is very important; besides it will adjust to isolate promastigote forms without contamination, and diagnosis will be made quickly and adequately. The analysis of the bone marrow smear and lymph node puncture will be useful for researches on amastigote forms.

The lack of sensitivity of anti-leishmanial antibodies may be demonstrated by IFAT, generally because of persistent antibodies or immunosupression, respectively. On the other side of time consumption in other diagnosis, such as blood culture and bone marrow and lymph node punctures, PCR is the most quick and reliable diagnostic test to this disease. However, this method can present some problems due to the genetic variability of different Leishmania strains or to PCR inhibition (15). 
A case of Canine Visceral Leishmaniasis in a female dog from Bocaina (São Paulo), with isolation of Leishmania promastigote forms from the bone marrow aspirate is presented bellow.

\section{CASE REPORT}

A Pit Bull female dog, aged one year and two months, weighing $20.6 \mathrm{~g}$, lived in Bauru until it was three months old and afterwards in Bocaina, São Paulo, Brazil. The animal presented weight loss, conjunctivitis, and nasal bleeding for two weeks, with several alopecia areas and intense exfoliation in the skin, muzzle and around the eyes, together with partial injury of the external ears (Figure 1 and Video 1), increased popliteal and pre-scapular lymph nodes, onychogryphosis and hepatosplenomegaly, normocytic and normochromic anemia, normal leucocytes with active monocytes and reactive lymphocytes, and high levels of creatinine and urea. Since the animal clinical presentation was compatible with leishmaniasis, serology was carried out by confirmation of suspicion. IFAT standardized by Camargo (4) was performed, and the animal presented a titer of 1280. Based on this result, immediately before euthanasia, the puncture of the right pre-scapular lymph node (Figure 2 and Video 2) was performed to study amastigote forms of Leishmania sp by aspirative cytology. Bone marrow puncture (Figure 3 and Video 3) was also performed at the iliac crest, and the obtained sample was inoculated in LIT (Liver Infusion Tryptose) medium and incubated in a stove at $28-30^{\circ} \mathrm{C}$. The lymph node aspirative cytologies, as well as the bone marrow aspirates, demonstrated several amastigote forms of Leishmania sp, such as amastigote nests, free in the intercellular space and in the macrophage cytoplasm (Figure 4). Leishmania sp was isolated from the bone marrow puncture after 13 days of incubation, and several promastigote forms, free and in rosette formation were visualized at a 1000X optical microscopy; the smears were stained by the Giemsa technique (Figure 5 and Video 4).

The PCR test was also performed for Leishmania sp from the bone marrow aspirate culture, using LINR4 and LIN19 primers (2), producing 720 base pairs, specific for Leishmania sp (Figure 6). 


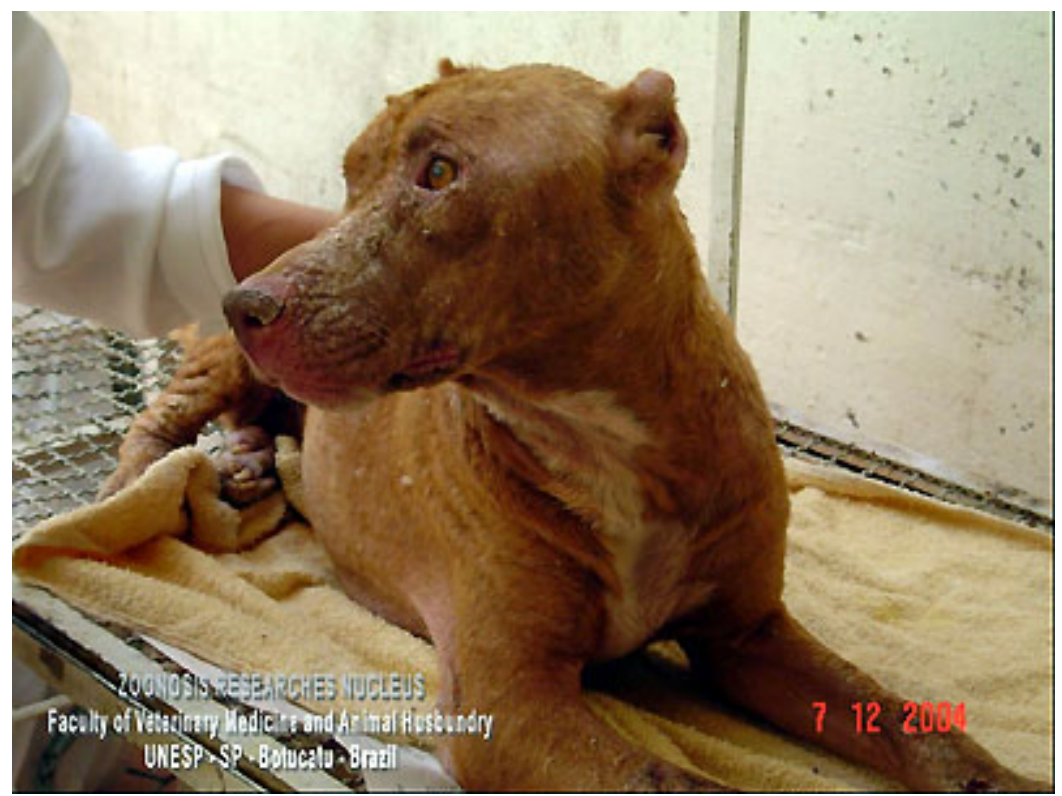

Figure 1: Clinical presentation of the dog, with generalized skin lesions in the body and partial damage of the external ears.

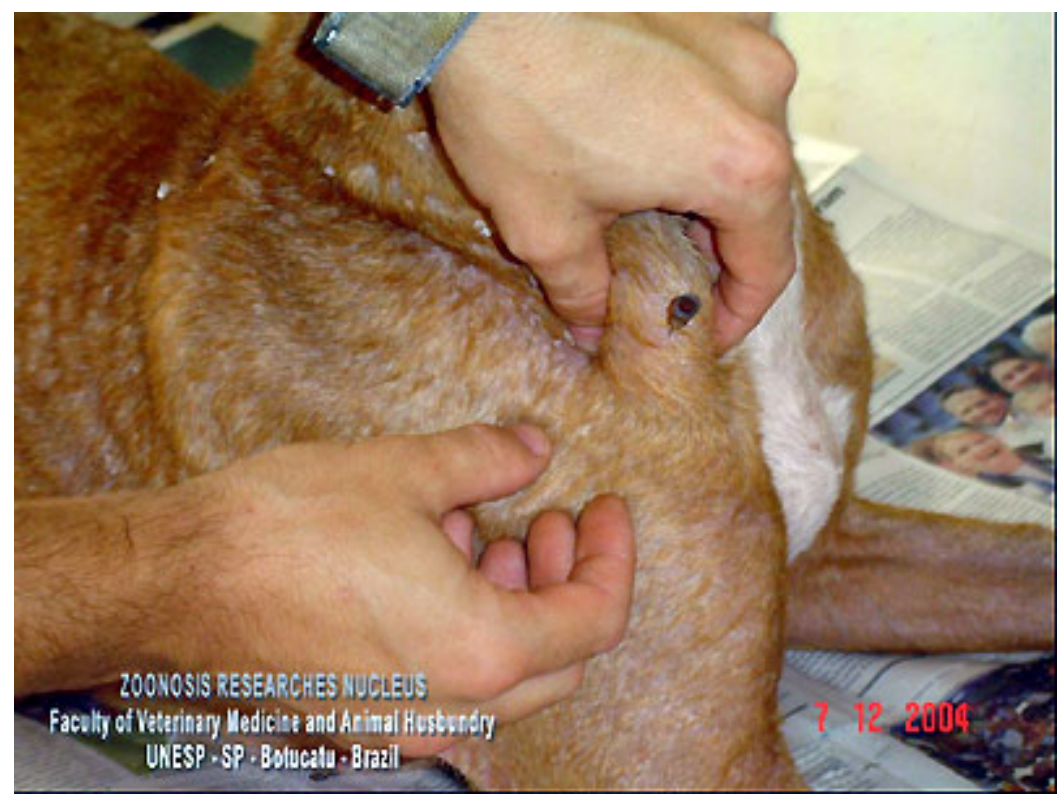

Figure 2: Puncture of the right pre-scapular lymph node 


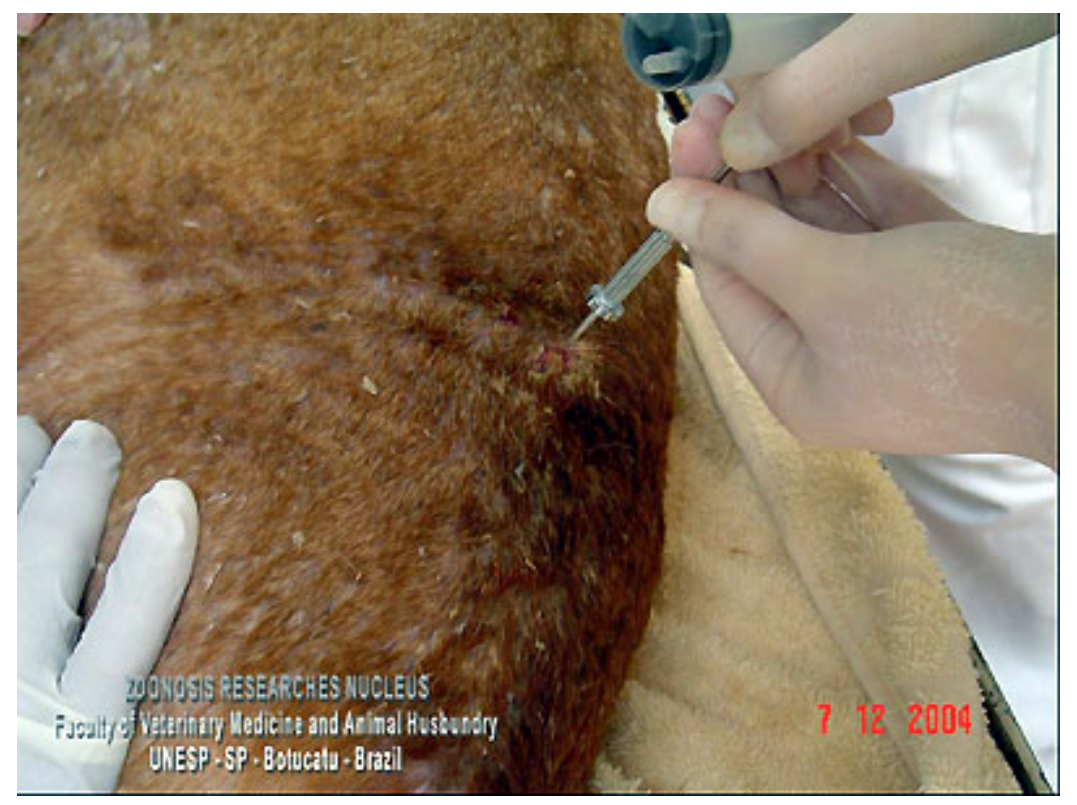

Figure 3: Puncture of the bone marrow

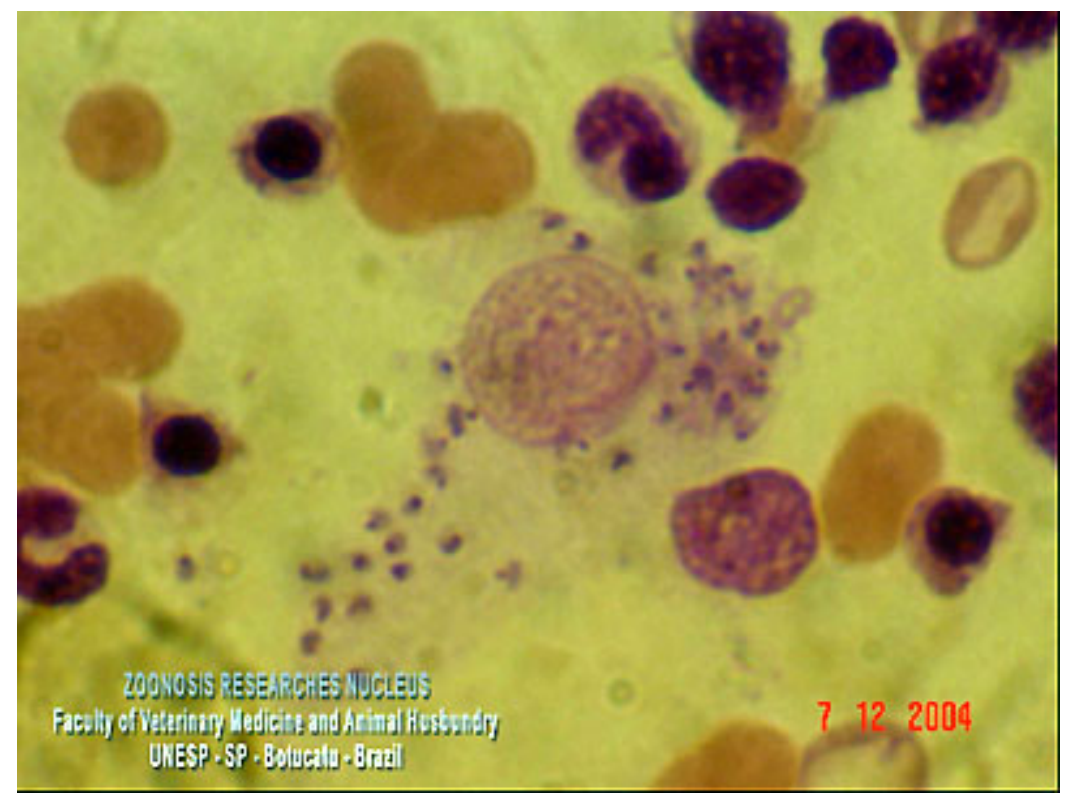

Figure 4: Amastigote forms of Leishmania sp from bone marrow aspirate, stained by the Giemsa method (X1000). 
H. Langoni et al. AMERICAN VISCERAL LEISHMANIASIS: A CASE REPORT. J. Venom. Anim. Toxins incl. Trop. Dis., 2005, 11, 3, p. 367

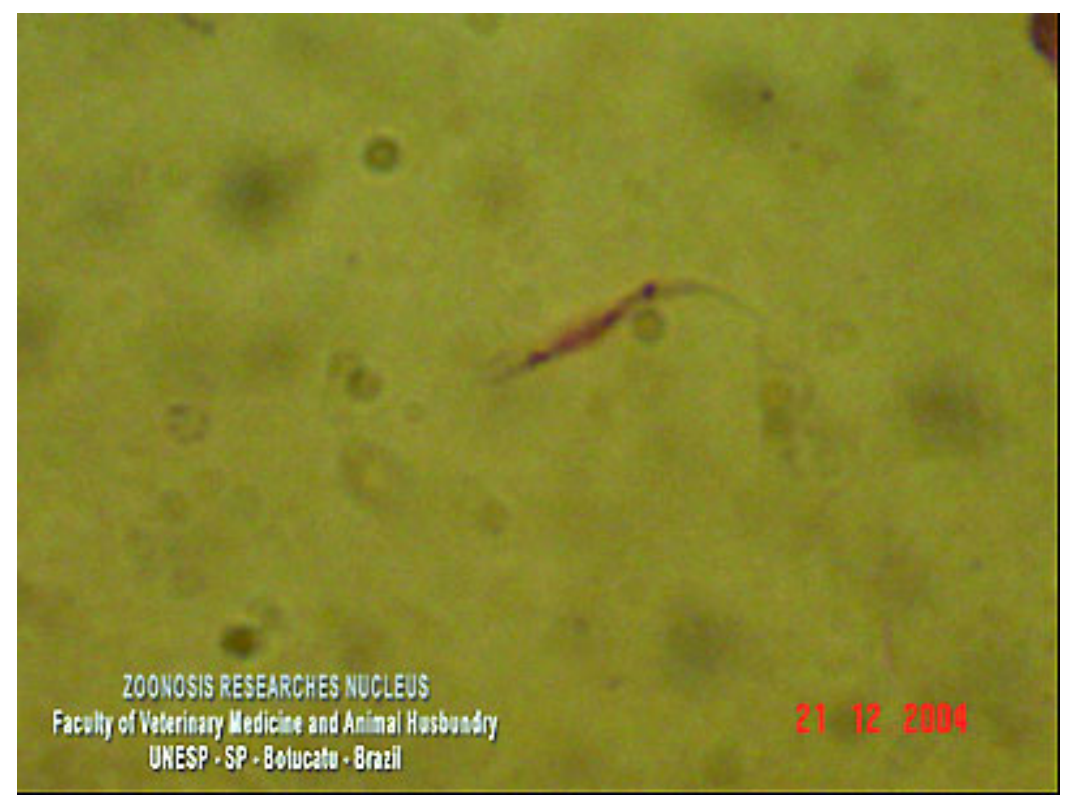

Figure 5: Smear of promastigote forms in LIT culture, isolated from the bone marrow puncture after 13 days of incubation at $28-30^{\circ} \mathrm{C}$, stained by the Giemsa method (x1000). 


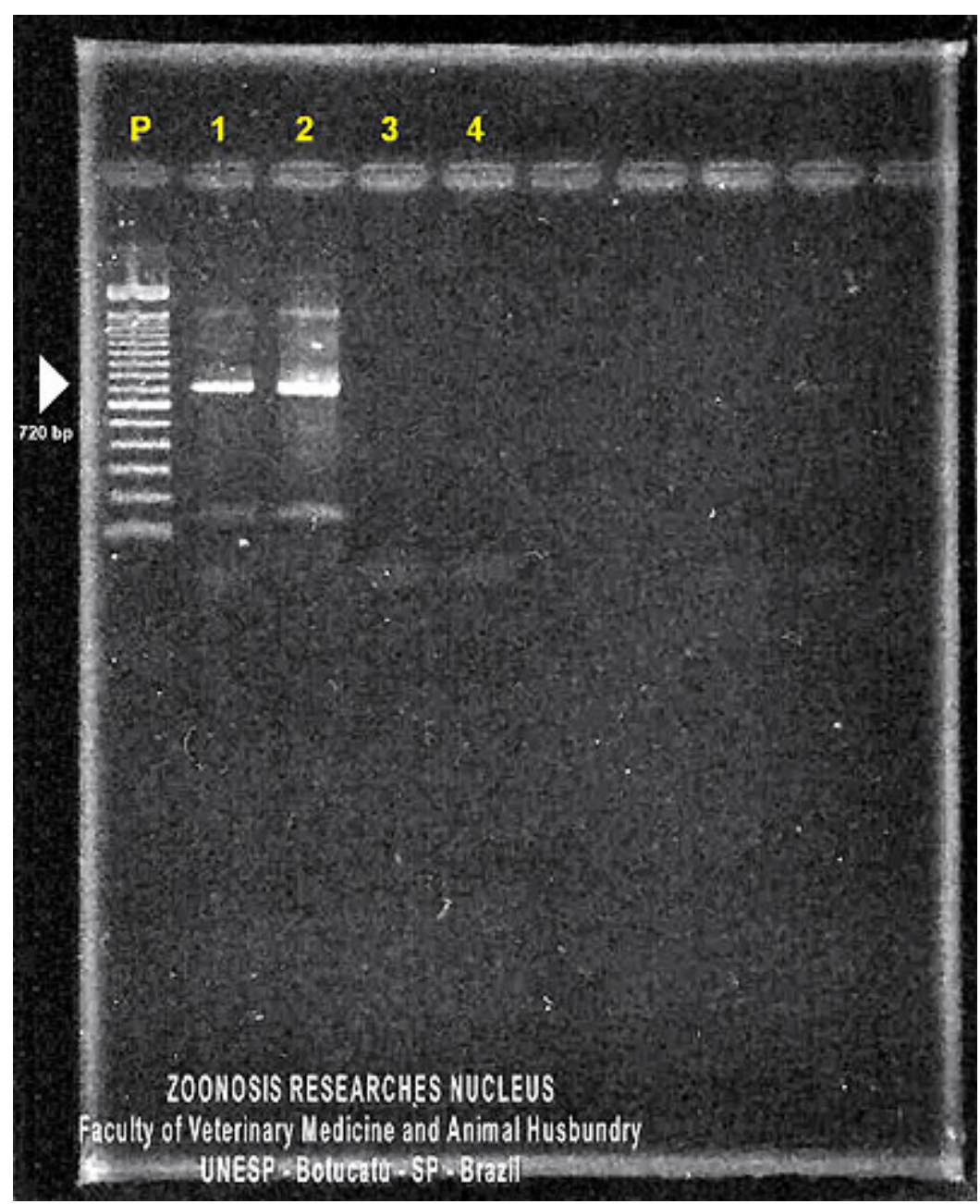

Figure 6: Result of the amplification of fragments of the KDNA minicircle of Leishmania $\mathrm{sp}$ by Polymerase Chain Reaction (PCR), obtained from the bone marrow aspirate culture. Electrophoresis on 1.5\% agarose gel stained with ethidium bromide showing a 720bp fragment derived from minicircles of Leishmania sp. P: molecular weight standard 100bp (100bp DNA ladder - Invitrogen ${ }^{\circledR}$ ); 1: positive control (Leishmania chagasi strain); 2: positive sample for Leishmania sp; 3: extraction buffer (EB); 4: negative control (Mix-PCR). 


\section{DISCUSSION}

When the subject is Canine Visceral Leishmaniasis, the diagnosis, several times, is a serious problem to veterinary medicine. There are many clinical signs, from apparently healthy animals to oligosymptomatic animals, and this confounds professionals, leading them to think it is another disease. There are animals in severe stages of the disease with characteristic signs, as the case reported. However, there are cases in which the disease remains unapparent for a long time, representing a public health problem (10).

Professionals and researchers need an appropriate, simple, sensitive, and specific method that leads to the diagnosis for not only the advanced cases, but also for the initial ones, oligosymptomatics and asymptomatics (1), because the recommendation is sacrifice in positive cases (Decree 51,838, on March 14 1963, article 9).

The clinical presentation of the disease changes from asymptomatic, self-limited infection to fatal Visceral Leishmaniasis. Canine Visceral Leishmaniasis incubation period lasts from few months to several years, depending on the parasite virulence and the host genetic susceptibility, demonstrated in humans and, experimentally, in animals (20). Dog infection by sandflies is followed by an asymptomatic phase, with anti-Leishmania antibodies present. These asymptomatic dogs are divided into two categories: those susceptible to the disease, which will be symptomatic and will probably die, and those resistant to the parasite (5).

Protozoan visualization may be possible from biopsia material or lymph node, bone marrow, liver or spleen puncture. The obtained material is used to make smear of digital impression in slides, histology, culture medium isolation or laboratory experimental animals inoculation. The specificity of these methods is $100 \%$ but sensitivity is variable, because protozoan distribution is not equal in the same tissue (10). The highest sensitivity (98\%) is reached when spleen puncture is utilized (11). The Indirect Fluorescent Antibody Test (IFAT), indicated by the Ministry of Health for diagnosis and epidemiologic Canine Visceral Leishmaniasis inquest, presents high sensitivity (90-100\%) and specificity of $80 \%$ to serum samples. Besides, it is known that identification of false-positive individuals in the IFAT test is due to cross reactions between Visceral Leishmaniasis and other two very important zoonosis: American Cutaneous Leishmaniasis and Chagas' disease (1).

DNA detection may be performed too, and in the case reported, bone marrow puncture was subjected to PCR for Leishmania $\mathrm{sp}$ detection. This test allows the 
identification and selective amplification of the parasite kinetoplast DNA. Unfortunately, there are many limitations in using this method in epidemiologic investigation due to the costs, reagents availability, equipments, and little adaptability of the test in the field (22); moreover, it is not always available to routine diagnoses. In conclusion, Canine Visceral Leishmaniasis diagnosis is indispensable, due not only to the public health impact the disease represents, but also to the need of establishing precise and quick diagnostic methods when we have oligosymptomatic and asymptomatic dogs, which represent extremely important reservoirs in the epidemiologic cycle of this zoonosis.

\section{REFERENCES}

1 ALVES WA., BEVILACQUA PD. Reflexões sobre a qualidade do diagnóstico da leishmaniose visceral canina em inquéritos epidemiológicos: o caso da epidemia de Belo Horizonte, Minas Gerais, Brasil, 1993-1997. Cad. Saúde Públ., 2004, 20, 259-65.

2 ARANSAY AM., SCOULICA E., TSELENTIS Y. Detection and identification of Leishmania DNA within naturally infected sand flies by seminested PCR on minicircle kinetoplastic DNA. Appl. Environ. Microbiol., 2000, 66, 1933-8.

3 ARIAS JR., MONTEIRO PS., ZICKER F. The reemergence of visceral leishmaniasis in Brazil. Emerg. Infect. Dis. 1996, 2, 145-6.

4 CAMARGO ME. Fluorescent antibody test for the serodiagnosis of American trypanosomiasis. Technical modification employing preserved culture forms of Trypanosoma cruzi in a slide test. Rev. Inst. Med.Trop. São Paulo, 1966, 8, 227-34.

5 DYE C., KILLICK-KENDRICK R., VITUTIA MM., WALTON R., KILLICK-KENDRICK M., HARITH AE., GUY MW., CANAVATI MC., HASIBEDER G. Epidemiology of canine leishmaniasis: prevalence, incidence and basic reproduction number calculated from a cross-sectional serological survey on the island of Gozo, Malta. Parasitology, 1992, 105, 35-41.

6 ENSERINK M. Infectious diseases. Has leishmaniasis become endemic in the U.S.? Science, 2000, 290, 1881-3. 
7 FEITOSA MM., IKEDA FA., LUVIZOTTO MCR., PERRI SHV. Clinical aspects from dogs naturally infected with visceral leishmaniasis in Araçatuba, São Paulo State, Brazil. Clinica Veterinaria, 2000, 28, 36-44.

8 FEITOSA MM. Leishmaniose visceral: um desafio crescente. São Paulo: Intervet pet, 2001. 15p.

9 GENARO O. Leishmaniose visceral americana. In: NEVES DP, MELO AL, GENARO O, LINARDI PM. Org. Parasitologia humana. Rio de Janeiro: Livraria Atheneu; 2000: 56-72.

10 GONTIJO CMF., MELO MN. Leishmaniose Visceral no Brasil: quadro atual, desafios e perspectivas. Rev. Bras. Epidemiol., 2004, 7, 338-49.

11 GUERIN PJ., OLLIARO P., SUNDAR S., BOELAERT M., CROFT SL., DESJEUX P., WASUNNA MK., BRYCESON ADM. Visceral leishmaniasis: current status of control, diagnosis, and treatment, and a proposed research and development agenda. Lancet Infect. Dis., 2002, 2, 494-501.

12 HARITH AE., KOLK AHJ., KAGER PA., LEEUWENBURG J., FABER FJ., MUIGAI R., KIUGU S., LAARMAN JJ. Evaluation of a newly developed direct agglutination test (DAT) for serodiagnosis and sero-epidemiological studies of visceral leishmaniasis: comparison with IFAT and ELISA. Trans. R. Soc. Trop. Med. Hyg., 1987, 81, 603-6.

13 HERWALDT BL. Leishmaniasis. Lancet, 1999, 354, 1191-9.

14 IKEDA FA., CIARLINI PC., FEITOSA MM., GONÇALVES ME., LUVIZOTTO MCR., DE LIMA VMF. Perfil hematológico de cães naturalmente infectados por Leishmania chagasi no município de Araçatuba - SP: um estudo retrospectivo de 191 casos. Clínica Veterinária, 2003, 47, 42-8.

15 IKONOMOPOULOS J., KOKOTAS S., GAZOULI M., ZAVRAS A., STOITSIOU M., GORGOULIS VG. Molecular diagnosis of leishmaniosis in dogs: comparative application of traditional diagnostic methods and the proposed assay on clinical samples. Vet. Parasitol., 2003, 113, 99-113.

16 KAR K. Serodiagnosis of leishmaniasis. Crit. Rev. Microbiol., 1995, 21, 123-52.

17 MOHAMMED AR., WRIGHT EP., ABDEL RAHMAN AM., KOLK A., LAARMAN JJ., PONDMAN KW. Serodiagnosis of Sudanese visceral and mucosal leishmaniasis: comparision of ELISA - immunofluorescence and indirect haemagglutination. Trans. R. Soc. Trop. Med. Hyg., 1986, 80, 271-4. 
18 MORENO J., ALVAR J. Canine leishmaniasis: epidemiological risk and the experimental model. Trends Parasitol., 2002,18, 399-405.

19 PEARSON RD., SOUSA AQ. Clinical spectrum of leishmaniasis. Clin. Infect. Dis., 1996, 22, 1-13.

20 REINER SL., LOCKSLEY RM. The regulation of immunity to Leishmania major. Ann. Rev. Immunol., 1995, 13, 151-77.

21 SILVA ES., GONTIJO CMF., PACHECO RS., FIÚZA VOP., BRAZIL RP. Visceral leishmaniasis in the Metropolitan Region of Belo Horizonte, State of Minas Gerais, Brazil. Mem. Inst. Oswaldo Cruz, 2001, 96, 285-91.

22 SILVA JCF. Leishmaniose visceral canina no município de Montes Claros, Minas Gerais, Brasil. Belo Horizonte: Instituto de Ciências Biológicas, Universidade Federal de Minas Gerais, 1997. 133 p. 